\title{
Examining the biomarkers and molecular mechanisms of medulloblastoma based on bioinformatics analysis
}

\author{
BIAO YANG ${ }^{*}$, JUN-XI DAI $^{*}$, YUAN-BO PAN, YAN-BIN MA and SHENG-HUACHU \\ Department of Neurosurgery, Shanghai Ninth People's Hospital Affiliated to Shanghai \\ Jiao Tong University School of Medicine, Shanghai 201999, P.R. China
}

Received February 28, 2018; Accepted April 2, 2019

DOI: $10.3892 / \mathrm{ol} .2019 .10314$

\begin{abstract}
Medulloblastoma (MB) is the most common malignant brain tumor in children. The aim of the present study was to predict biomarkers and reveal their potential molecular mechanisms in MB. The gene expression profiles of GSE35493, GSE50161, GSE74195 and GSE86574 were downloaded from the Gene Expression Omnibus (GEO) database. Using the Limma package in $\mathrm{R}$, a total of 1,006 overlapped differentially expressed genes (DEGs) with the cut-off criteria of $\mathrm{P}<0.05$ and $\mid \log _{2}$ fold-change $(\mathrm{FC}) \mid>1$ were identified between MB and normal samples, including 540 upregulated and 466 downregulated genes. Furthermore, the Gene Ontology (GO) and the Kyoto Encyclopedia of Genes and Genomes (KEGG) pathway enrichment analysis were also performed using the Database for Annotation, Visualization and Integrated Discovery (DAVID) online tool to analyze functional and pathway enrichment. The Search Tool for Retrieval of Interacting Genes database was subsequently used to construct a protein-protein interaction (PPI) network and the network was visualized in Cytoscape. The top 11 hub genes, including CDK1, CCNB1, CCNB2, PLK1, CDC20, MAD2L1, AURKB, CENPE, TOP2A, KIF2C and PCNA, were identified from the PPI network. The survival
\end{abstract}

Correspondence to: $\mathrm{Dr}$ Sheng-Hua Chu, Department of Neurosurgery, Shanghai Ninth People's Hospital Affiliated to Shanghai Jiao Tong University School of Medicine, 280 Mohe Road, Baoshan, Shanghai 201999, P.R. China

E-mail: shenghuachu@126.com

*Contributed equally

Abbreviations: MB, medulloblastoma; GEO, Gene Expression Omnibus; DEGs, differentially expressed genes; DAVID, Database for Annotation, Visualization and Integrated Discovery; GO, Gene Ontology; KEGG, Kyoto Encyclopedia of Genes and Genomes; PPI, protein-protein interaction; STRING, Search Tool for the Retrieval of Interacting Genes; MCODE, Molecular Complex Detection; BP, biological process; $\mathrm{MF}$, molecular function; $\mathrm{CC}$, cell component

Key words: medulloblastoma, bioinformatics, differentially expressed genes, biomarkers, modules curves for hub genes in the dataset GSE85217 predicted the association between the genes and survival of patients with MB. The top 3 modules were identified by the Molecular Complex Detection plugin. The results indicated that the pathways of DEGs in module 1 were primarily enriched in cell cycle, progesterone-mediated oocyte maturation and oocyte meiosis; and the most significant functional pathways in modules 2 and 3 were primarily enriched in mismatch repair and ubiquitin-mediated proteolysis, respectively. These results may help elucidate the pathogenesis and design novel treatments for MB.

\section{Introduction}

Medulloblastoma (MB) is the most common solid tumor in children, comprising $15-20 \%$ of pediatric central nervous system tumors $(1,2)$. MB may occur at all ages, however its peak incidence is between 4 and 7 years (3). In 2016, the World Health Organization classified MB into four subtypes, including WNT-activated, SHH-activated, group 3 and group 4, by combining molecular profiling with histology (4). In addition, as these tumors occur in the posterior fossa, clinical symptoms are often too vague for accurate and prompt diagnosis (5). The therapeutic options include maximal safe surgical resection, radiation and chemotherapy (3). However, cerebellar mutism may occur in $>25 \%$ of the cases following maximal surgical resection in patients with high-risk MB; recovering patients may still experience dysarthria and neurocognitive dysfunction (6). In addition, adjuvant chemotherapy and radiotherapy may lead to hearing loss and the development of secondary tumors (6). The main cause of mortality in MB is metastatic disease, which is unresectable (7). Although multimodal therapy significantly improves the prognosis of MB, approximately one-third of the patients eventually succumb to the disease (3). Therefore, further research on the underlying molecular mechanisms is imperative, in order to design more efficient and precise treatment strategies to improve patient survival.

With the completion of the Human Genome Project, molecular diagnosis and therapy have become available in clinical practice, which is helpful for improving the accuracy and efficacy of diagnosis and treatment (4). Using bioinformatics and microarray analysis, it is possible to further examine the underlying gene characteristics and molecular 
mechanisms involved in the proliferation, invasion and metastasis of MB $(8,9)$. For example, mitotic kinases and WEE1 G2 checkpoint kinase were identified as rational therapeutic targets for $\mathrm{MB}$ by performing an integrated genomic analysis using structural and functional methods (10).

In the present study, 4 databases with 115 samples of $\mathrm{MB}$ and normal tissues were downloaded from the Gene Expression Omnibus (GEO) database. Following screening of differentially expressed genes (DEGs) though package Limma in R, Gene Ontology (GO) and Kyoto Encyclopedia of Genes and Genomes (KEGG) analyses were performed to analyze the potential functional and pathway enrichment, and a protein-protein interaction (PPI) network was subsequently constructed using the Search Tool for Retrieval of Interacting Genes (STRING) database and visualized with Cytoscape software, in order to identify biomarkers and examine the potential underlying molecular mechanisms in MB. Therefore, the results of the present study may improve our understanding of MB, identify potential biomarkers and indicate methods of diagnosis and treatment for future research.

\section{Materials and methods}

Microarray data. In the present study, the gene expression profiles of GSE35493 (11), GSE50161 (12), GSE74195 (13) and GSE86574 (https://www.ncbi.nlm.nih.gov/geo/query/ acc.cgi?acc=GSE86574) were downloaded from the GEO database (http://www.ncbi.nlm.nih.gov/geo/). A total of 115 samples, including $78 \mathrm{MB}$ and 37 normal samples, had been hybridized on the Affymetrix Human Genome U133 Plus 2.0 Array (HG-U133_Plus_2) on the GPL570 platform.GSE35493 included $17 \mathrm{MB}$ and 9 normal samples, GSE50161 included $22 \mathrm{MB}$ and 13 normal samples, GSE74195 included $23 \mathrm{MB}$ and 5 normal samples, and GSE86574 included $16 \mathrm{MB}$ and 10 normal samples. GSE85217 from the GPL22286 platform included $613 \mathrm{MB}$ samples.

Identification of DEGs. The raw data in CEL files were transformed into gene symbols based on the downloaded platform annotation files. The data were preprocessed, including background correction, normalization and summarization, via R 3.4.1 software (https://www.r-project.org/) (14). Following robust multiarray average normalization, Limma in $\mathrm{R}$ package (version 3.26.9) was used to screen DEGs (15). The genes meeting the cut-off criteria of adjusted $\mathrm{P}<0.05$ and $\mid \log _{2}$ fold-change $(\mathrm{FC}) \mid>1$ were selected as the DEGs.

Functional and pathway enrichment analysis. After acquiring the DEGs, GO enrichment analysis and KEGG pathway enrichment analysis were performed through DAVID (https:// david.ncifcrf.gov/) online tool to identify functional categories of DEGs (16). GO analysis of DEGs included biological process (BP), molecular function (MF) and cell component (CC). In addition, the terms with $\mathrm{P}<0.05$ were considered to indicate a statistically significant difference.

Construction of the PPI network and selection of modules. To identify hub genes and screen modules, the DEGs were uploaded to STRING (version 10.5; http://www.string-db.org/) to analyze and set up the PPI network (17). Subsequently, the

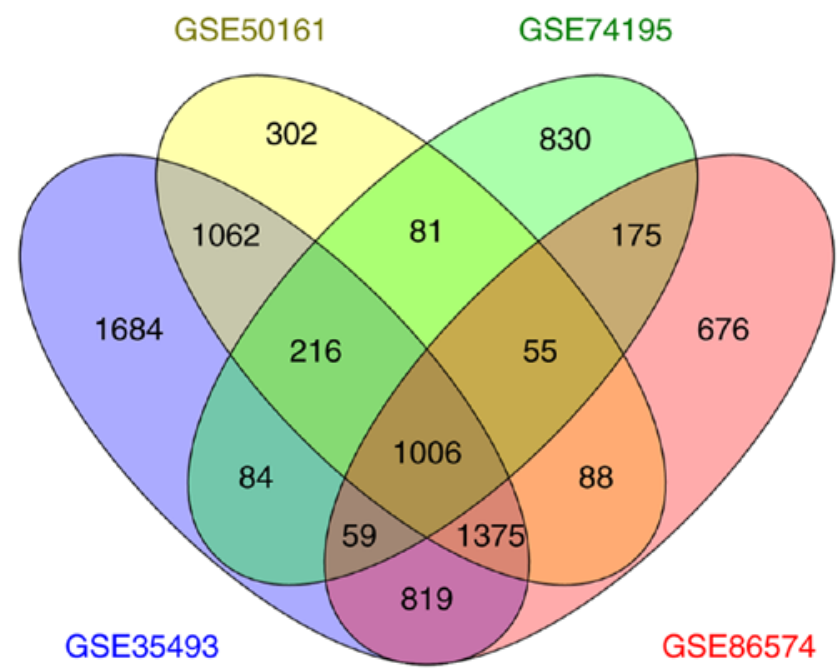

Figure 1. Venn diagram of differentially expressed genes among the 4 datasets.

network was visualized in Cytoscape (version 3.5.1; www. cytoscape.org) (18). The Cytoscape software was applied to search for hub genes with CytoHubba, a plugin to hub an object from complex networks, and modules with Molecular Complex Detection (MCODE) $(19,20)$. Furthermore, hub genes in selected modules were analyzed via DAVID to examine pathway enrichment.

Survival analysis. To assess the association between hub genes and survival, $613 \mathrm{MB}$ samples with clinical data from GSE85217 were selected and survival curves were drawn by recruiting the survival package in $R$.

\section{Results}

Identification of DEGs. Basing on the cut-off criteria of $\mathrm{P}<0.05$ and $\log _{2}$ fold-change (FC) $\mid>1$, a total of $6,305,4,185$, 2,506 and 4,253 DEGs between MB and normal samples were screened from GSE35493, GSE50161, GSE74195 and GSE86574, respectively. DEGs from GSE35493 included 5,242 upregulated and 1,063 downregulated genes. DEGs from GSE50161 included 3,025 upregulated and 1,160 downregulated genes. DEGs from GSE74195 included 1,315 upregulated and 1,191 downregulated genes. DEGs from GSE86574 included 2,772 upregulated and 1,481 downregulated genes. In addition, a total of 1,006 mutual DEGs were screened, among those 4 datasets, by performing a Venn diagram analysis (Fig. 1), where 540 were upregulated and 466 were downregulated.

Functional and pathway enrichment analysis. Submitting 1,006 mutual DEGs to DAVID provided further insight into the function of these DEGs and the molecular mechanisms implicated in MB. The top 10 significant terms of each GO category (Table I) and the top 10 terms of KEGG category (Table II) were selected. The GO analysis results demonstrated that overlapped DEGs were significantly associated with cell division, mitotic nuclear division and chemical synaptic transmission in the BP category; cell junction, condensed chromosome kinetochore and postsynaptic membrane in the 
Table I. Top 10 significant GO terms of BP, MF and CC.

\begin{tabular}{|c|c|c|c|c|}
\hline Category & Term & Description & Count & P-value \\
\hline $\mathrm{BP}$ & GO:0051301 & Cell division & 77 & $1.54 \times 10^{-27}$ \\
\hline $\mathrm{BP}$ & GO:0007067 & Mitotic nuclear division & 55 & $6.14 \times 10^{-20}$ \\
\hline $\mathrm{BP}$ & GO:0007268 & Chemical synaptic transmission & 45 & $1.47 \times 10^{-13}$ \\
\hline $\mathrm{BP}$ & GO:0000082 & G1/S transition of mitotic cell cycle & 28 & $1.02 \times 10^{-12}$ \\
\hline $\mathrm{BP}$ & GO:0007062 & Sister chromatid cohesion & 27 & $8.76 \times 10^{-12}$ \\
\hline $\mathrm{BP}$ & GO:0006260 & DNA replication & 33 & $1.23 \times 10^{-11}$ \\
\hline $\mathrm{BP}$ & GO:0000070 & Mitotic sister chromatid segregation & 12 & $1.48 \times 10^{-08}$ \\
\hline $\mathrm{BP}$ & GO:0007059 & Chromosome segregation & 18 & $4.14 \times 10^{-08}$ \\
\hline $\mathrm{BP}$ & GO:0000086 & G2/M transition of mitotic cell cycle & 24 & $5.11 \times 10^{-07}$ \\
\hline $\mathrm{BP}$ & GO:0007076 & Mitotic chromosome condensation & 8 & $4.23 \times 10^{-06}$ \\
\hline $\mathrm{CC}$ & GO:0030054 & Cell junction & 65 & $8.14 \times 10^{-14}$ \\
\hline $\mathrm{CC}$ & GO:0000777 & Condensed chromosome kinetochore & 24 & $2.74 \times 10^{-11}$ \\
\hline $\mathrm{CC}$ & GO:0045211 & Postsynaptic membrane & 37 & $8.92 \times 10^{-11}$ \\
\hline $\mathrm{CC}$ & GO:0014069 & Postsynaptic density & 34 & $1.40 \times 10^{-10}$ \\
\hline $\mathrm{CC}$ & GO:0005654 & Nucleoplasm & 206 & $2.20 \times 10^{-09}$ \\
\hline $\mathrm{CC}$ & GO:0030425 & Dendrite & 43 & $3.92 \times 10^{-08}$ \\
\hline $\mathrm{CC}$ & GO:0000775 & Chromosome, centromeric region & 16 & $8.32 \times 10^{-08}$ \\
\hline $\mathrm{CC}$ & GO:0030496 & Midbody & 24 & $9.65 \times 10^{-08}$ \\
\hline $\mathrm{CC}$ & GO:0043025 & Neuronal cell body & 40 & $1.67 \times 10^{-07}$ \\
\hline $\mathrm{CC}$ & GO:0005874 & Microtubule & 39 & $3.78 \times 10^{-07}$ \\
\hline MF & GO:0005515 & Protein binding & 531 & $3.92 \times 10^{-09}$ \\
\hline MF & GO:0008017 & Microtubule binding & 32 & $7.31 \times 10^{-08}$ \\
\hline MF & GO:0003682 & Chromatin binding & 40 & $4.88 \times 10^{-05}$ \\
\hline MF & GO:0005524 & ATP binding & 110 & $7.45 \times 10^{-05}$ \\
\hline $\mathrm{MF}$ & GO:0019901 & Protein kinase binding & 38 & $1.00 \times 10^{-04}$ \\
\hline $\mathrm{MF}$ & GO:0005201 & Extracellular matrix structural constituent & 13 & $1.27 \times 10^{-04}$ \\
\hline MF & GO:0005509 & Calcium ion binding & 60 & $1.87 \times 10^{-04}$ \\
\hline MF & GO:0017075 & Syntaxin-1 binding & 7 & $1.88 \times 10^{-04}$ \\
\hline MF & GO:0004890 & GABA-A receptor activity & 7 & $2.63 \times 10^{-04}$ \\
\hline $\mathrm{MF}$ & GO:0005219 & Ryanodine-sensitive calcium-release channel activity & 4 & $5.07 \times 10^{-04}$ \\
\hline
\end{tabular}

GO, Gene Ontology; BP, biological process; MF, molecular function; CC, cell component.

$\mathrm{CC}$ category; and protein binding, microtubule binding and chromatin binding in the MF category $(\mathrm{P}<0.05)$ (Table I). In addition, the enriched KEGG pathway analysis results primarily included cell cycle, DNA replication and retrograde endocannabinoid signaling (Table II).

Module screening from the PPI network. Among the 4 datasets, the overlapped 1,006 DEGs were analyzed with the PPI network via STRING, and interaction with a score $>0.9$ was subsequently obtained in the following analysis. Furthermore, the hub genes with degrees $>44$ were screened out based on CytoHubba. In total, 11 nodes were screened out as hub genes, including CDK1 (degree=90), CCNB1 (degree=68), CCNB2 (degree=60), PLK1 (degree=60), CDC20 (degree=57), MAD2L1 (degree=53), AURKB (degree=50), CENPE (degree=46), TOP2A (degree=45), KIF2C (degree=45) and PCNA (degree=45; Fig. 2A). Among the 11 hub genes, the node with the highest degree (90) was CDK1. Additionally, four heat maps of the expression of 11 hub genes in GSE35493, GSE50161, GSE74195 and GSE86574 are presented in Fig. 3.

Furthermore, following MCODE analysis, 7 modules were identified to be available and the top 3 significant modules are presented in Fig. 2B-D. Furthermore, the top 3 pathways in each module are listed in Table III. Module 1 had 29 nodes, 404 edges and the highest score (score=28.857). In this module, the top 3 enriched KEGG pathways were cell cycle, progesterone-mediated oocyte maturation, and oocyte meiosis. In addition, module 2 (score $=11.6$ ) had 31 nodes and 174 edges, and the pathways were primarily associated with mismatch repair, DNA replication and nucleotide excision repair. Module 3 (score $=10$ ) had 10 nodes and 45 edges, which were significantly enriched in ubiquitin-mediated proteolysis $(\mathrm{P}<0.05$; Table III).

Survival analysis. The survival curves for hub genes in the dataset GSE85217 are presented in Fig. 4. The expressions of CCNB1 $(\mathrm{P}=0.03108), \mathrm{CCNB} 2(\mathrm{P}=0.00336), \mathrm{CDC} 20$ 
Table II. Top 10 significant Kyoto Encyclopedia of Genes and Genomes pathways.

\begin{tabular}{|c|c|c|c|c|}
\hline Term & Description & Count & P-value & Genes \\
\hline hsa04110 & Cell cycle & 28 & $3.95 \times 10^{-11}$ & $\begin{array}{l}\text { E2F5, DBF4, TTK, CHEK1, PTTG1, CHEK2, CCNE2, CDC45, } \\
\text { MCM7, CDKN2C, CDK1, ESPL1, CDK6, CDC20, MCM2, } \\
\text { CDK4, MCM3, WEE1, CDC25A, MCM5, CCNB1, MAD2L1, } \\
\text { CCNB2, CCND2, PLK1, PCNA, BUB1B, ABL1 }\end{array}$ \\
\hline hsa03030 & DNA replication & 14 & $7.26 \times 10^{-09}$ & $\begin{array}{l}\text { MCM2, RNASEH2A, MCM3, MCM5, PRIM1, RFC3, RFC4, } \\
\text { MCM7, POLE2, RFC2, POLD1, PRIM2, PCNA, FEN1 }\end{array}$ \\
\hline hsa04723 & $\begin{array}{l}\text { Retrograde } \\
\text { endocannabinoid } \\
\text { signaling }\end{array}$ & 22 & $1.50 \times 10^{-08}$ & $\begin{array}{l}\text { GABRD, GABRG1, GABRA2, GABRA1, GNAI3, GABRA4, } \\
\text { GABRB2, GABRB1, GNG13, MAPK10, GRIA4, RIMS1, } \\
\text { KCNJ3, ITPR1, SLC17A7, SLC32A1, KCNJ6, KCNJ9, GRIA1, } \\
\text { MGLL, GNG3, GNG4 }\end{array}$ \\
\hline hsa04727 & GABAergic synapse & 20 & $2.14 \times 10^{-08}$ & $\begin{array}{l}\text { GABRD, GABRG1, GABRA2, GABARAPL1, GABRA1, } \\
\text { GNAI3, SLC6A1, GABRA4, GABRB2, GABRB1, GABBR1, } \\
\text { GNG13, GABBR2, GLS2, SLC32A1, KCNJ6, ABAT, GNG3, } \\
\text { GNG4, GAD1 }\end{array}$ \\
\hline hsa05032 & Morphine addiction & 19 & $3.66 \times 10^{-07}$ & $\begin{array}{l}\text { GABRD, GABRG1, GABRA2, GNAI3, GABRA1, GABRA4, } \\
\text { GABRB2, GABRB1, GABBR1, GNG13, GABBR2, KCNJ3, } \\
\text { ADORA1, SLC32A1, KCNJ6, KCNJ9, PDE1A, GNG3, GNG4 }\end{array}$ \\
\hline hsa05033 & Nicotine addiction & 12 & $2.42 \times 10^{-06}$ & $\begin{array}{l}\text { SLC17A7, GABRD, SLC32A1, GABRG1, GABRA2, GABRA1, } \\
\text { GABRA4, GRIA1, GABRB2, GABRB1, GRIN2A, GRIA4 }\end{array}$ \\
\hline hsa04728 & Dopaminergic synapse & 20 & $1.55 \times 10^{-05}$ & $\begin{array}{l}\text { SCN1A, CALY, PPP2R3A, GNAI3, KIF5A, GRIN2A, GNG13, } \\
\text { MAPK10, GRIA4, KCNJ3, ITPR1, KCNJ6, KCNJ9, PPP1R1B, } \\
\text { GRIA1, CREB3L4, CAMK2B, GNG3, PPP3CA, GNG4 }\end{array}$ \\
\hline hsa04713 & Circadian entrainment & 16 & $5.91 \times 10^{-05}$ & $\begin{array}{l}\text { GNAI3, GRIN2A, GNG13, GRIA4, KCNJ3, ITPR1, KCNJ6, } \\
\text { KCNJ9, GRIA1, RYR3, RYR1, RYR2, CAMK2B, GUCY1B3, } \\
\text { GNG3, GNG4 }\end{array}$ \\
\hline hsa03430 & Mismatch repair & 8 & $8.58 \times 10^{-05}$ & EXO1, MSH6, RFC3, RFC4, RFC2, MSH2, POLD1, PCNA \\
\hline hsa04115 & p53 signaling pathway & 13 & $9.34 \times 10^{-05}$ & $\begin{array}{l}\text { CCNB1, CCNE2, CDK1, TP53I3, CCNB2, CCND2, RRM2, } \\
\text { SIAH1, CHEK1, CDK6, CHEK2, CDK4, GTSE1 }\end{array}$ \\
\hline
\end{tabular}

( $\mathrm{P}=0.026), \mathrm{KIF} 2 \mathrm{C}(\mathrm{P}=0.01622)$, MAD2L1 $(\mathrm{P}=0.00145), \mathrm{PLK} 1$ $(\mathrm{P}=0.00325)$ and TOP2A $(\mathrm{P}=0.01387)$ were negatively associated with patient survival time.

\section{Discussion}

Identifying the molecular mechanisms of MB, which has unique gene expression signatures, is of critical importance for targeted diagnosis and treatment (21). In the present study, $78 \mathrm{MB}$ and 37 normal samples were collected from the GEO database for bioinformatics analysis, aiming to identify hidden biomarkers and elucidate the molecular mechanisms in MB. A total of 1,006 mutual DEGs were screened from the four microarray datasets GSE35493, GSE50161, GSE74195 and GSE86574 using the Limma in R package.

The GO analysis results of the DEGs revealed that the overlapped DEGs were primarily associated with mitosis, including cell division, mitotic nuclear division, $\mathrm{G}_{1} / \mathrm{S}$ transition of the mitotic cell cycle, sister chromatid cohesion, sister chromatid segregation, chromosome segregation, $\mathrm{G}_{2} / \mathrm{M}$ transition of the mitotic cell cycle, and mitotic chromosome condensation in the BP category. In addition, Aurora kinase $B$ regulates multiple stages of mitosis, and its inhibitors may inhibit the growth of Group 3 MBs and prolong survival (22). These results suggested that it may be possible to treat MB by regulating the key biomarkers of mitosis $(23,24)$. In addition, it was also observed that these genes were enriched in cell junction, condensed chromosome kinetochore, protein binding, microtubule binding and chromatin binding. Certain RNA binding proteins, including MSI1, DDX3X and CCAR1, were reported to play important roles in the growth and/or maintenance of MB (25).

Following KEGG pathway analysis, the genes were found to be significantly associated with cell cycle, DNA replication, retrograde endocannabinoid signaling, GABAergic synapse, morphine addiction, nicotine addiction, dopaminergic synapse, circadian entrainment, mismatch repair, and p53 signaling pathway. A previous study reported that the defect of NEO1, which was necessary for cell cycle progression, arrests cells at the $G_{2} / M$ phase in $M B(26)$. These results indicated that cannabinoids, morphine and nicotine, consistent with previous studies, were likely associated with the progression of brain tumors (27-29). Therefore, these pathway analysis results may enable the prediction of novel therapeutic targets.

Furthermore, the top 11 hub genes, including CDK1, CCNB1, CCNB2, PLK1, CDC20, MAD2L1, AURKB, 
A

KCNJ10
TIA1 GEMIN4 NXJURP DDX39A KPNA2 KIFC2 FGF1 KSR2 GABRA1 ITPR1 GRIN2A GRA GRIA4 NRXIN2

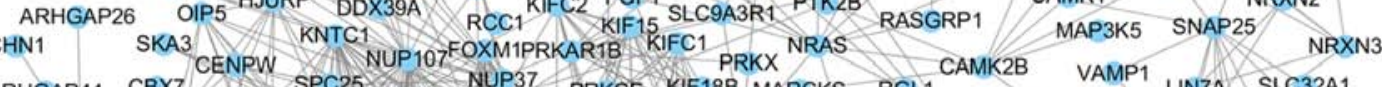
ARHGAP44 CBX7 CENPW SPC25 107 NUP37 PRKGE KIF18B MARCKS RGL1 PAMK2B VAMP1 LIN7A SLC32A1 DEPDC1B
RHOBTB1 MIS18A ASPM CENPF SMN CENPM EIF2C KIF5A EZR SPRY2 ARHGDIG TACC3 RACGAP1 CENPH. ESPL1 BUB1B NCAPD KIF4A KIF14EPHB2 SGIP1 KIAA0319 PACSIN1 RIMS1 CARNS1 TUBB HAUS1 ZWILCH. DCC ZWINT CCNB) NDC80 PRC1 KIF26A SSRP1 REPS2 EPS15 PPP2R3A GAD1 CNDP1 RUVBL1 HMX2 TTK CENPE CDCA8 CDC25A EDKN2C SH3G2 EPHA4 DNAJC6 CPLX1 ABAT

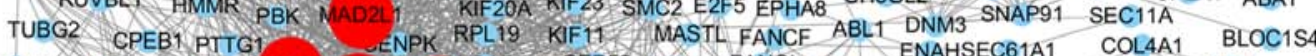
STMN4 TUBB4A NEDD1 ECNB? IIRKB GAP5 CEP55 GDKN3 FANCI UBE2T ENAHSEC61A1 COL4A1 ALPL

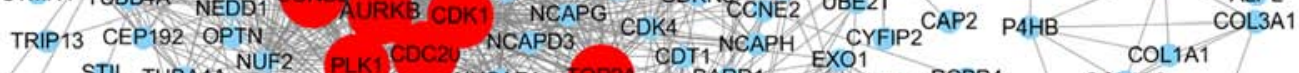

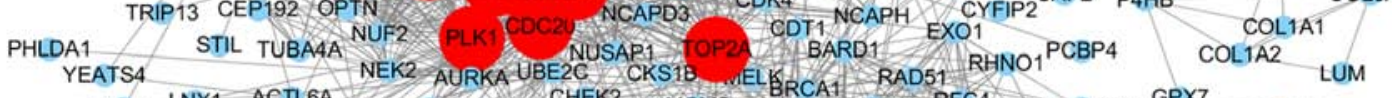

PTMA LNX1 ACTLEA NCAPG2 CHEK2 MCM5 BRCA1 RAD51 RFC4 RAD51AP1 GPX7 LUM SLC25A4 FBXW7 SIAH1 ECT2 UBE2S CDC45 CKS2 MNB PRIM1 CHEK1. RFC3 BRIP1 CYP2J2 SNCA GTSE1 FBXL3 LRR1 RRM2 MCMT MCM2 PCNA RAD54L SOD2 GSTP1 KLHL2 TRIM9 $28 T B 16$ SMARCE1 M KIAA0101. MCM3 RFC2 POLD1 MSH6 RBBP8
UBE2E2 BE2E2 SMARCC1 GINS1 EEN1 MCM1OMSH2 HMGN1 TDG HMGB2
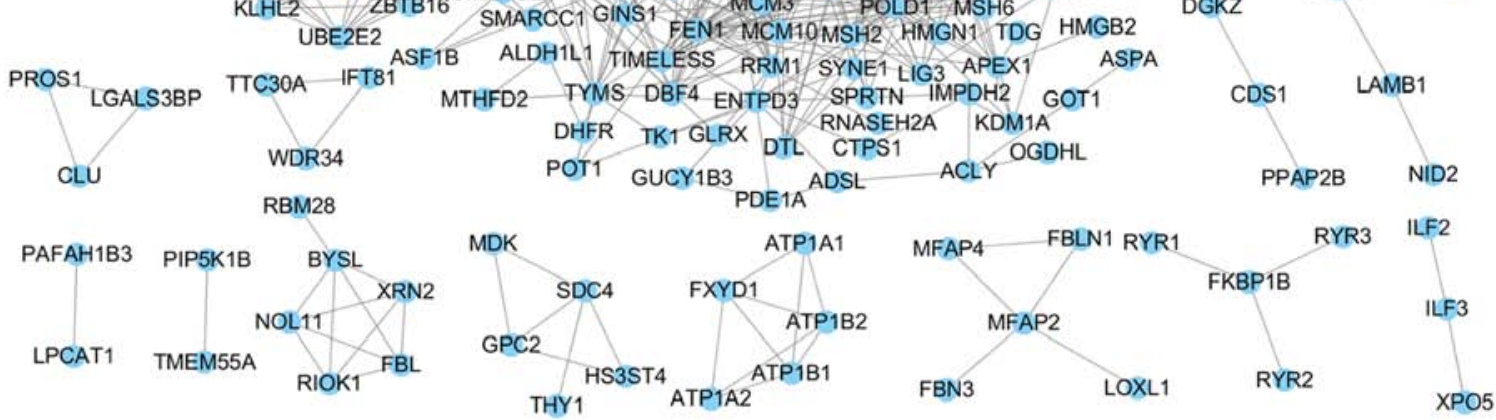

PVALB

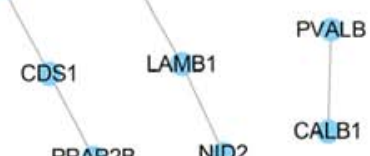

CALB1

PANX1

P2RX7

XPO5 P2RX5

ADAM11 ALDH1A1 TMOD1 BMPR1B RYK SEMA3A EPB49 TAF1A TPRKB BCL6 CDH10 MGLL ZNF217 VPS37B NTRK2 DTX4

LGI1 HSD17B6MYBPC1 CHRDL1 VANGL2 DPYSL3 ADD3 DEK TP53RK BCOR JUP AKR1A1 FAM60A FAM125A DYNLT1 DNER
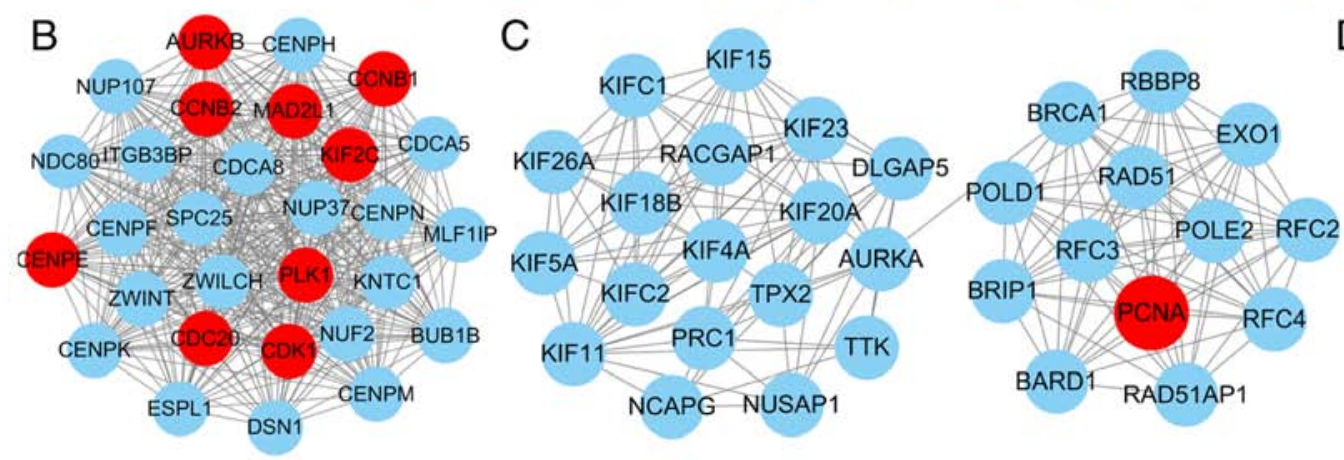

D

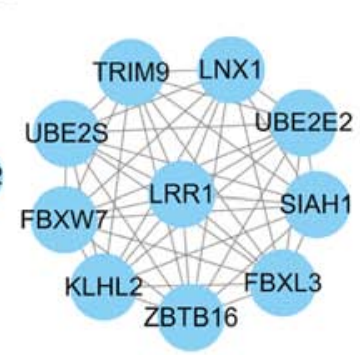

Figure 2. PPI network and modules of DEGs in MB. (A) PPI network. (B) Module 1. (C) Module 2. (D) Module 3. The circular nodes represent DEGs. The edges/lines stand for the regulatory association between two nodes. The top 11 hub genes are highlighted with red circles. PPI, protein-protein interaction; DEGs, differentially expressed genes; MB, medulloblastoma.

CENPE, TOP2A, KIF2C and PCNA, were identified using the PPI network. It was reported that inhibiting the catalytic activity of CDK1 using VMY-1-103 may severely disrupt the mitotic cycle of MB cells (24). It was previously reported that the combined expression of MYC, LDHB and CCNB1 as potential prognostic biomarkers may predict survival and provide a more accurate basis for the targeted therapy of patients with MB (30). The inhibitors of PLK1, an oncogenic kinase that controls cell cycle and proliferation, inhibited mitosis in MB cells, and patients expressing high levels of PLK1 were considered as high-risk. All findings indicated that PLK1 is a possible therapeutic target for patients with MB $(31,32)$. In another study, it was reported that the expression levels of TOP2A may be a potential biomarker for 
Table III. Top 10 significant KEGG pathways of the DEGs in top 3 modules.

\begin{tabular}{lclccl}
\hline Module & Term & \multicolumn{1}{c}{ KEGG names } & Count & P-value & \multicolumn{1}{c}{ Genes } \\
\hline Module 1 & hsa04110 & Cell cycle & 8 & $5.80 \times 10^{-11}$ & CCNB1, CDK1, CDC20, CCNB2, PLK1, \\
& hsa04914 & $\begin{array}{l}\text { Progesterone-mediated } \\
\text { oocyte maturation }\end{array}$ & 5 & $4.65 \times 10^{-06}$ & CCNB1, CDK1, PLK1, MAD2L1, CCNB2, \\
& hsa04114 & Oocyte meiosis & 5 & $1.14 \times 10^{-05}$ & CDK1, MAD2L1, PLK1, CDC20, ESPL1 \\
Module 2 & hsa03430 & Mismatch repair & 6 & $3.25 \times 10^{-10}$ & EXO1, RFC3, RFC4, RFC2, POLD1, PCNA \\
& hsa03030 & DNA replication & 6 & $3.59 \times 10^{-09}$ & RFC3, RFC4, POLE2, RFC2, POLD1, PCNA \\
hodule 3 & hsa03420 & Nucleotide excision repair & 6 & $1.45 \times 10^{-08}$ & RFC3, RFC4, POLE2, RFC2, POLD1, PCNA \\
& hbiquitin mediated proteolysis & 4 & $7.41 \times 10^{-05}$ & FBXW7, SIAH1, UBE2S, UBE2E2 \\
\hline
\end{tabular}

KEGG, Kyoto Encyclopedia of Genes and Genomes; DEGs, differentially expressed genes.
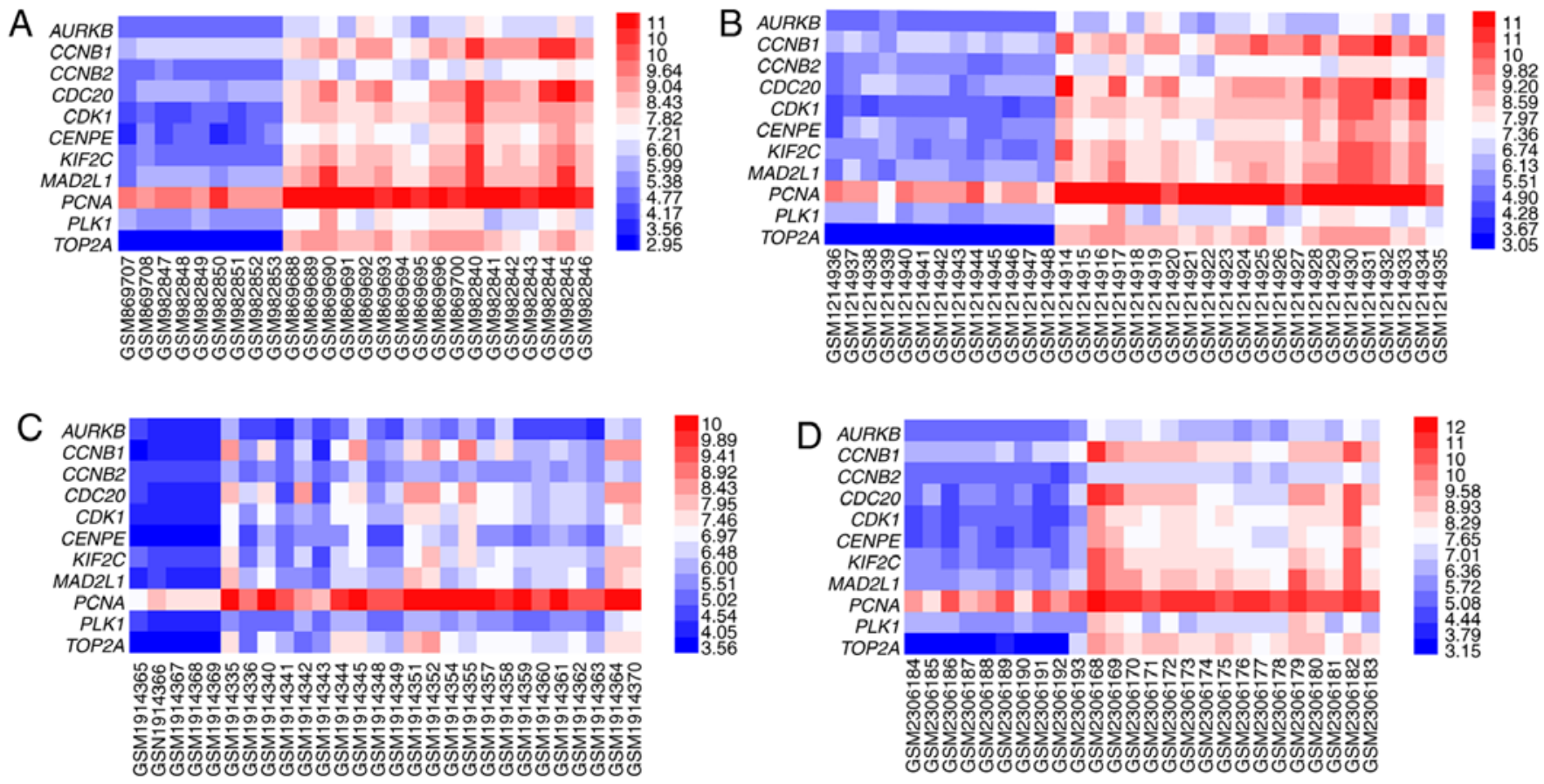

Figure 3. Hub gene expression heat maps among the 4 databases. (A) Hub gene expression heat map of GSE35493. (B) Hub gene expression heat map of GSE50161. (C) Hub gene expression heat map of GSE74195. (D) Hub gene expression heat map of GSE86574. Red, upregulation; blue, downregulation.

sensitivity to etoposide in patients with MB (33). PCNA, which may be used to demonstrate the proliferative phase of the cell cycle, was significantly associated with MB grade, suggesting that PCNA may be a biomarker for assessing grade and the possibility of recurrence in MB (34).

However, 6 hub genes, including CCNB2, CDC20, MAD2L1, AURKB, CENPE and KIF2C, have yet to be verified, to the best of our knowledge, in MB by systematic searches through PubMed, there were a number of studies on other tumors, particularly brain tumors, including glioma (33-44). It was reported that CDC20 was a critical regulator of tumor-initiating cell proliferation and survival of glioblastoma cells (35). Combining the findings of other studies, CDC20 was found to play a role in cell cycle progression, apoptosis and brain development, and these findings indicated that it may be a potential novel target for therapeutic intervention in brain tumors, particularly MB (36,37). In addition, the RNA levels of CDC20 and MAD2L1 were associated with glioma grade, which suggested a clinical benefit as a biomarker (38). Other studies demonstrated that MAD2L1 was of diagnostic value in several tumors, including salivary duct carcinomas, breast cancer and acute lymphoblastic leukemia (39-41). Furthermore, CCNB2 was predicted as a tumor-associated factor similar to CCNB1 (42). AURKA was reported to be the target of some molecule inhibitors, such as BMS-754807 and SIX3, aiming to inhibit diffuse intrinsic pontine glioma or astrocytoma $(43,44)$. The knockdown of CENPE, which is highly expressed in pediatric glioma, combined with temozolomide treatment, was found to lead to inhibition of glioma 

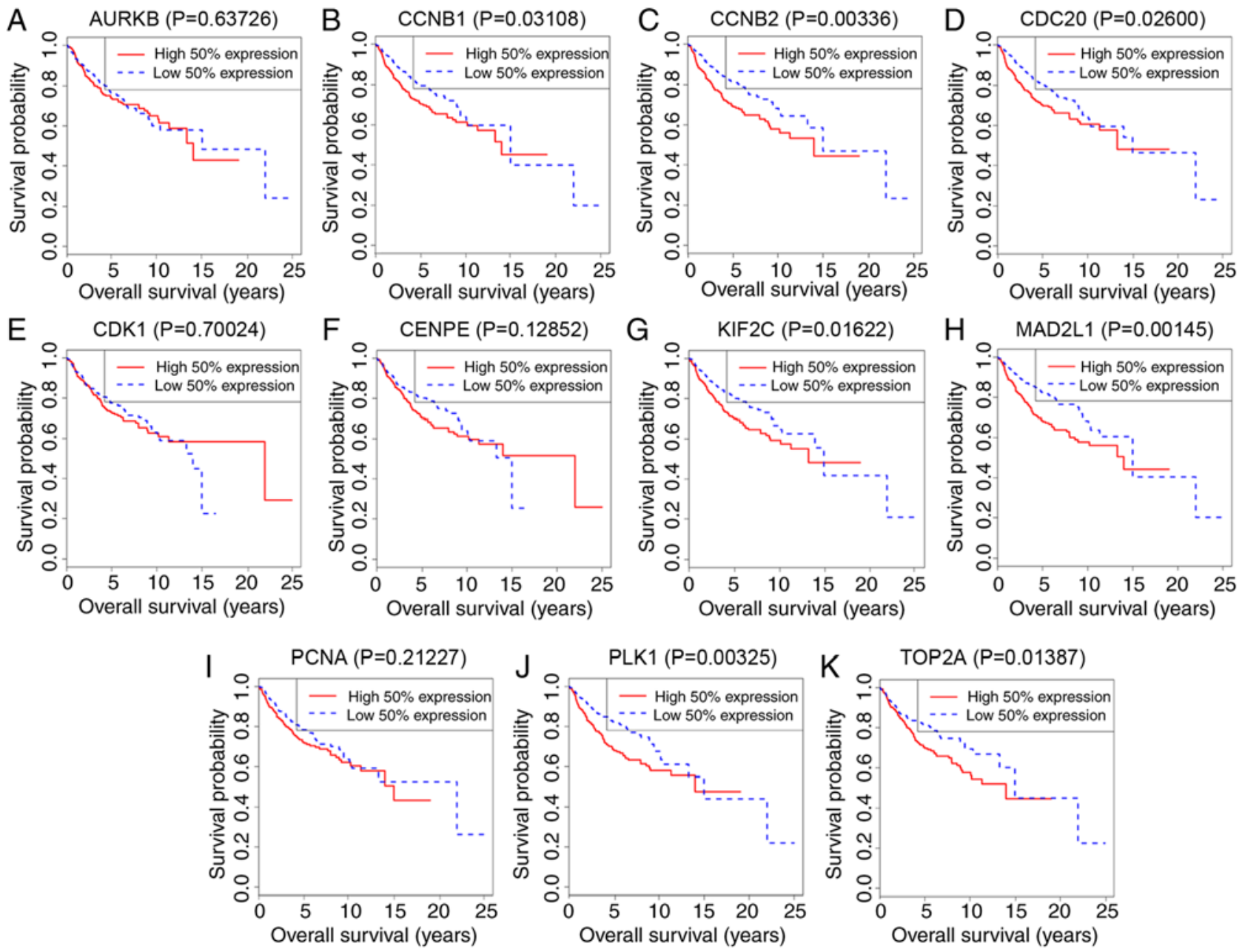

Figure 4. Survival analysis for hub genes in the dataset GSE85217. The difference in survival between low and high expression of (A) AURKB (P=0.63726), (B) CCNB1 ( $\mathrm{P}=0.03108)$, (C) CCNB2 ( $\mathrm{P}=0.00336)$, (D) CDC20 ( $\mathrm{P}=0.02600)$, (E) CDK1 ( $\mathrm{P}=0.70024),(\mathrm{F})$ CENPE ( $\mathrm{P}=0.12852),(\mathrm{G}) \mathrm{KIF} 2 \mathrm{C}$ ( $\mathrm{P}=0.01622)$, (H) MAD2L1 ( $\mathrm{P}=0.00145)$, (I) PCNA $(\mathrm{P}=0.21227)$, (J) PLK1 $(\mathrm{P}=0.00325)$ and $(\mathrm{K})$ TOP2A $(\mathrm{P}=0.01387)$. Red lines represent high expression and blue lines represent low expression of the hub genes.

cell proliferation (45). KIF2C was reported to be associated with histopathological glioma grades, which indicated this gene may be a potential biomarker for prognosis in patients with glioma (46). Taken together, although their significance has yet to been confirmed, to the best of our knowledge, these findings suggest that the 6 hub genes may be potential biomarkers in the diagnosis, treatment and prognosis of MB.

In conclusion, the findings of the present study provided an integrated bioinformatics analysis of 1,006 overlapped DEGs that may be involved in the growth, recurrence and metastasis of MB. A total of 11 hub genes, including CDK1, CCNB1, CCNB2, PLK1, CDC20, MAD2L1, AURKB, CENPE, TOP2A, KIF2C and PCNA, were identified as novel potential biomarkers. These findings may provide further insight into the underlying molecular mechanisms and identify novel biomarkers for evaluating the diagnosis and prognosis, and advance the treatment of MB. However, further molecular biological research is required to confirm the clinical value of our findings.

\section{Acknowledgements}

Not applicable.

\section{Funding}

The present study was supported by the Scientific Research Foundation for the Returned Overseas Chinese Scholars (082003), the Research Foundation of Shanghai Municipal Health and Family Planning Commission (201540266), Shanghai Jiao Tong University Medicine-Engineering Cross Research Foundation (YG2015MS25) and the Research Foundation of Shanghai No. 3 People's Hospital Affiliated to Shanghai Jiao Tong University School of Medicine (syz2015-015).

\section{Availability of data and materials}

The datasets used and/or analyzed during the present study were obtained from the GEO, DAVID and STRING databases.

\section{Authors' contributions}

SHC and BY were involved in the conception and design of the research and drafting the manuscript. YBP participated in the acquisition of data. BY and JXD performed the analysis 
and interpretation of data. YBM was involved in the statistical analysis. All authors read and approved the final manuscript.

\section{Ethics approval and consent to participate}

Not applicable.

\section{Patient consent for publication}

Not applicable.

\section{Competing interests}

The authors declare that they have no competing interests.

\section{References}

1. Gilbertson RJ: Medulloblastoma: Signalling a change in treatment. Lancet Oncol 5: 209-218, 2004.

2. Bartlett F, Kortmann R and Saran F: Medulloblastoma. Clin Oncol (R Coll Radiol) 25: 36-45, 2013.

3. Gerber NU, Mynarek M, von Hoff K, Friedrich C, Resch A and Rutkowski S: Recent developments and current concepts in medulloblastoma. Cancer Treat Rev 40: 356-365, 2014.

4. Louis DN, Perry A, Reifenberger G, von Deimling A, Figarella-Branger D, Cavenee WK, Ohgaki H, Wiestler OD, Kleihues P and Ellison DW: The 2016 World health organization classification of tumors of the central nervous system: A summary. Acta Neuropathol 131: 803-820, 2016.

5. Quinlan A and Rizzolo D: Understanding medulloblastoma. JAAPA 30: 30-36, 2017.

6. Sengupta S, Pomeranz Krummel D and Pomeroy S: The evolution of medulloblastoma therapy to personalized medicine F1000 Res 6: 490, 2017.

7. Archer TC, Mahoney EL and Pomeroy SL: Medulloblastoma: Molecular classification-based personal therapeutics. Neurotherapeutics 14: 265-273, 2017.

8. Shou Y, Robinson DM, Amakye DD, Rose KL, Cho YJ, Ligon KL, Sharp T, Haider AS, Bandaru R, Ando Y, et al: A five-gene hedgehog signature developed as a patient preselection tool for hedgehog inhibitor therapy in medulloblastoma. Clin Cancer Res 21: 585-593, 2015.

9. Khatua S and Zaky W: The biologic era of childhood medulloblastoma and clues to novel therapies. Future Oncol 10: 637-645, 2014.

10. Harris PS, Venkataraman S, Alimova I, Birks DK, Balakrishnan I, Cristiano B, Donson AM, Dubuc AM, Taylor MD, Foreman NK, et al: Integrated genomic analysis identifies the mitotic checkpoint kinase WEE1 as a novel therapeutic target in medulloblastoma. Mol Cancer 13: 72, 2014.

11. Birks DK, Donson AM, Patel PR, Sufit A, Algar EM, Dunham C, Kleinschmidt-DeMasters BK, Handler MH, Vibhakar R and Foreman NK: Pediatric rhabdoid tumors of kidney and brain show many differences in gene expression but share dysregulation of cell cycle and epigenetic effector genes. Pediatr Blood Cancer 60: 1095-1102, 2013

12. Griesinger AM, Birks DK, Donson AM, Amani V, Hoffman LM, Waziri A, Wang M, Handler MH and Foreman NK Characterization of distinct immunophenotypes across pediatric brain tumor types. J Immunol 191: 4880-4888, 2013

13. de Bont JM, Kros JM, Passier MM, Reddingius RE, Sillevis Smitt PA, Luider TM, den Boer ML and Pieters R: Differential expression and prognostic significance of SOX genes in pediatric medulloblastoma and ependymoma identified by microarray analysis. Neuro Oncol 10: 648-660, 2008.

14. Gautier L, Cope L, Bolstad BM and Irizarry RA: Affy-analysis of Affymetrix GeneChip data at the probe level. Bioinformatics 20 : 307-315, 2004.

15. Ritchie ME, Phipson B, Wu D, Hu Y, Law CW, Shi W and Smyth GK: limma powers differential expression analyses for RNA-sequencing and microarray studies. Nucleic Acids Res 43: e 47,2015 .

16. Huang da W, Sherman BT and Lempicki RA: Systematic and integrative analysis of large gene lists using DAVID bioinformatics resources. Nat Protoc 4: 44-57, 2009.
17. Jensen LJ, Kuhn M, Stark M, Chaffron S, Creevey C, Muller J, Doerks T, Julien P, Roth A, Simonovic M, et al: STRING 8-a global view on proteins and their functional interactions in 630 organisms. Nucleic Acids Res 37: D412-416, 2009.

18. Smoot ME, Ono K, Ruscheinski J, Wang PL and Ideker T: Cytoscape 2.8: New features for data integration and network visualization. Bioinformatics 27: 431-432, 2011

19. Chin CH, Chen SH, Wu HH, Ho CW, Ko MT and Lin CY: cytoHubba: Identifying hub objects and sub-networks from complex interactome. BMC Syst Biol 4 (Suppl 8): S11, 2014.

20. Bader GD and Hogue CW: An automated method for finding molecular complexes in large protein interaction networks. BMC Bioinformatics 4: 2, 2003.

21. Brandes AA, Bartolotti M, Marucci G, Ghimenton C, Agati R, Fioravanti A, Mascarin M, Volpin L, Ammannati F, Masotto B, et al: New perspectives in the treatment of adult medulloblastoma in the era of molecular oncology. Crit Rev Oncol Hematol 94: 348-359, 2015.

22. Diaz RJ, Golbourn B, Faria C, Picard D, Shih D, Raynaud D, Leadly M,MacKenzie D, Bryant M,Bebenek M, et al: Mechanism of action and therapeutic efficacy of Aurora kinase B inhibition in MYC overexpressing medulloblastoma. Oncotarget 6: 3359-3374, 2015

23. Alimova I, Ng J, Harris P, Birks D, Donson A, Taylor MD, Foreman NK, Venkataraman S and Vibhakar R: MPS1 kinase as a potential therapeutic target in medulloblastoma. Oncol Rep 36: 2633-2640, 2016.

24. Ringer L, Sirajuddin P, Heckler M, Ghosh A, Suprynowicz F, Yenugonda VM, Brown ML, Toretsky JA, Uren A, Lee Y, et al: VMY-1-103 is a novel CDK inhibitor that disrupts chromosome organization and delays metaphase progression in medulloblastoma cells. Cancer Biol Ther 12: 818-826, 2011.

25. Bish R and Vogel C: RNA binding protein-mediated post-transcriptional gene regulation in medulloblastoma. Mol Cells 37: 357-364, 2014

26. Milla LA, Arros A, Espinoza N, Remke M, Kool M, Taylor MD, Pfister SM, Wainwright BJ and Palma V: Neogenin1 is a sonic hedgehog target in medulloblastoma and is necessary for cell cycle progression. Int J Cancer 134: 21-31, 2014.

27. Ellert-Miklaszewska A, Grajkowska W, Gabrusiewicz K, Kaminska B and Konarska L: Distinctive pattern of cannabinoid receptor type II (CB2) expression in adult and pediatric brain tumors. Brain Res 1137: 161-169, 2007.

28. Sepsova V, Krusek J, Zdarova Karasova J, Zemek F, Musilek K, Kuca K and Soukup O: The interaction of quaternary reversible acetylcholinesterase inhibitors with the nicotinic receptor. Physiol Res 63: 771-777, 2014.

29. Sardi I, la Marca G, Giovannini MG, Malvagia S, Guerrini R, Genitori L, Massimino M and Arico M: Detection of doxorubicin hydrochloride accumulation in the rat brain after morphine treatment by mass spectrometry. Cancer Chemother Pharmacol 67: 1333-1340, 2011 .

30. de Haas T, Hasselt N, Troost D, Caron H, Popovic M, Zadravec-Zaletel L, Grajkowska W, Perek M, Osterheld MC, Ellison D, et al: Molecular risk stratification of medulloblastoma patients based on immunohistochemical analysis of MYC, LDHB, and CCNB1 expression. Clin Cancer Res 14: 4154-4160, 2008.

31. Pezuk JA, Brassesco MS, Ramos PMM, Scrideli CA and Tone LG: Polo-like kinase 1 pharmacological inhibition as monotherapy or in combination: Comparative effects of polo-like kinase 1 inhibition in medulloblastoma cells. Anticancer Agents Med Chem 17: 1278-1291, 2017.

32. Triscott J, Lee C, Foster C, Manoranjan B, Pambid MR, Berns R, Fotovati A, Venugopal C, O'Halloran K, Narendran A, et al: Personalizing the treatment of pediatric medulloblastoma: Polo-like kinase 1 as a molecular target in high-risk children. Cancer Res 73: 6734-6744, 2013.

33. Uesaka T, Shono T, Kuga D, Suzuki SO, Niiro H, Miyamoto K, Matsumoto K, Mizoguchi M, Ohta M, Iwaki T and Sasaki T: Enhanced expression of DNA topoisomerase II genes in human medulloblastoma and its possible association with etoposide sensitivity. J Neurooncol 84: 119-129, 2007.

34. Kayaselcuk F, Zorludemir S, Gumurduhu D, Zeren H and Erman T: PCNA and Ki-67 in central nervous system tumors: Correlation with the histological type and grade. J Neurooncol 57: $115-121,2002$.

35. Xie Q, Wu Q, Mack SC, Yang K, Kim L, Hubert CG, Flavahan WA, Chu C, Bao S and Rich JN: CDC20 maintains tumor initiating cells. Oncotarget 6: 13241-13254, 2015. 
36. Wang L, Zhang J, Wan L, Zhou X, Wang Z and Wei W: Targeting $\mathrm{Cdc} 20$ as a novel cancer therapeutic strategy. Pharmacol Ther 151: 141-151, 2015.

37. Ji P, Zhou X, Liu Q, Fuller GN, Phillips LM and Zhang W: Driver or passenger effects of augmented c-Myc and Cdc20 in gliomagenesis. Oncotarget 7: 23521-23529, 2016.

38. Bie L, Zhao G, Cheng P, Rondeau G, Porwollik S, Ju Y, Xia XQ and McClelland M: The accuracy of survival time prediction for patients with glioma is improved by measuring mitotic spindle checkpoint gene expression. PLoS One 6: e25631, 2011.

39. Ko YH, Roh JH, Son YI, Chung MK, Jang JY, Byun H, Baek CH and Jeong HS: Expression of mitotic checkpoint proteins BUB1B and MAD2L1 in salivary duct carcinomas. J Oral Pathol Med 39: 349-355, 2010.

40. Krapf G, Kaindl U, Kilbey A, Fuka G, Inthal A, Joas R, Mann G, Neil JC, Haas OA and Panzer-Grumayer ER: ETV6/RUNX1 abrogates mitotic checkpoint function and targets its key player MAD2L1. Oncogene 29: 3307-3312, 2010.

41. Wang Z, Katsaros D, Shen Y, Fu Y, Canuto EM, Benedetto C, $\mathrm{Lu} \mathrm{L}$, Chu WM, Risch HA and Yu H: Biological and clinical significance of MAD2L1 and BUB1, genes frequently appearing in expression signatures for breast cancer prognosis. PLoS One 10: e0136246, 2015.

42. Manni I, Mazzaro G, Gurtner A, Mantovani R, Haugwitz U, Krause K, Engeland K, Sacchi A, Soddu S and Piaggio G: NF-Y mediates the transcriptional inhibition of the cyclin B1, cyclin B2, and cdc25C promoters upon induced G2 arrest. J Biol Chem 276: $5570-5576,2001$
43. Halvorson KG, Barton KL, Schroeder K, Misuraca KL, Hoeman C, Chung A, Crabtree DM, Cordero FJ, Singh R, Spasojevic I, et al: A high-throughput in vitro drug screen in a genetically engineered mouse model of diffuse intrinsic pontine glioma identifies BMS-754807 as a promising therapeutic agent. PLoS One 10: e0118926, 2015.

44. Yu Z, Sun Y, She X, Wang Z, Chen S, Deng Z, Zhang Y, Liu Q, Liu Q, Zhao C, et al: SIX3, a tumor suppressor, inhibits astrocytoma tumorigenesis by transcriptional repression of AURKA/B J Hematol Oncol 10: 115, 2017.

45. Liang ML, Hsieh TH, Ng KH, Tsai YN, Tsai CF, Chao ME, Liu DJ, Chu SS, Chen W, Liu YR, et al: Downregulation of miR-137 and miR-6500-3p promotes cell proliferation in pediatric high-grade gliomas. Oncotarget 7: 19723-19737, 2016.

46. Bie L, Zhao G, Wang YP and Zhang B: Kinesin family member $2 \mathrm{C}$ (KIF2C/MCAK) is a novel marker for prognosis in human gliomas. Clin Neurol Neurosurg 114: 356-360, 2012

(i) (2) This work is licensed under a Creative Commons Attribution-NonCommercial-NoDerivatives 4.0 International (CC BY-NC-ND 4.0) License. 